\title{
Meta-analysis: insulin sensitizers for the treatment of non-alcoholic steatohepatitis
}

\author{
M. O. Rakoski , A. G. Singal*, M. A. M. Rogers ${ }^{\dagger} \&$ H. Conjeevaram*
}

*Department of Internal Medicine,

Division of Gastroenterology,

University of Michigan Medical

Center, Ann Arbor, MI, USA.

†Division of General Medicine,

University of Michigan Medical

Center, Ann Arbor, MI, USA.

\section{Correspondence to:}

Dr H. Conjeevaram, Division of Gastroenterology, University of Michigan Medical Center, 3912

Taubman Center, SPC 5362,

Ann Arbor, MI 48109, USA

E-mail: omsairam@med.umich.edu

\section{Publication data}

Submitted 20 June 2010

First decision 12 July 2010

Resubmitted 31 August 2010

Accepted 1 September 2010

EV Pub Online 23 September 2010

As part of AP\&T's peer-review process, a technical check of this meta-analysis was performed by Dr. P. Collins.

\section{SUMMARY}

\section{Background}

Non-alcoholic fatty liver disease generally has a benign course; however, patients with non-alcoholic steatohepatitis (NASH) may progress to cirrhosis and hepatocellular carcinoma. Currently, there is a lack of consensus about optimal NASH treatment.

\section{Aim}

To assess the efficacy of insulin-sensitizing agents on histological and biochemical outcomes in randomized control trials of biopsy-proven NASH.

\section{Methods}

Multiple online databases and conference abstracts were searched. Random effects meta-analyses were performed, with assessment for heterogeneity and publication bias.

\section{Results}

Nine trials were included; five trials using thiazolidinediones (glitazones), three using metformin and one trial using both drugs. There was no publication bias. Compared with controls, glitazones resulted in improved steatosis $(\mathrm{WMD}=0.57,95 \%$ CI $0.36-0.77, P=<0.001$ ), hepatocyte ballooning $(\mathrm{WMD}=0.36,95 \%$ CI $0.24-0.49, P<0.001)$ and ALT $(\mathrm{WMD}=16.4,95 \%$ CI 7.7-25.0, $P<0.001)$, but not inflammation $(P=0.09)$ or fibrosis $(P=0.11)$. In patients without diabetes, glitazones significantly improved all histological and biochemical outcomes, most importantly including fibrosis (WMD $=0.29,95 \%$ CI $0.078-0.51, P=0.008$ ). Metformin failed to improve any pooled outcome.

\section{Conclusions}

Treatment of NASH with glitazones, but not metformin, demonstrates a significant histological and biochemical benefit, especially in patients without diabetes. Additional studies are needed to investigate long-term outcomes of glitazone therapy in patients without diabetes.

Aliment Pharmacol Ther 2010; 32: 1211-1221 


\section{INTRODUCTION}

Non-alcoholic fatty liver disease (NAFLD) is a growing health concern in the United States. It is the most common cause of liver disease, afflicting up to $30-40 \%$ of the general population and $60-91 \%$ of patients with underlying diabetes or morbid obesity. ${ }^{1-4}$ NAFLD has been shown to be an independent risk factor for increased cardiovascular morbidity and mortality. ${ }^{5-8}$ Whereas the majority of patients with NAFLD have simple steatosis that has a benign course, about $20 \%$ of patients will have a more severe form known as non-alcoholic steatohepatis (NASH), a histological diagnosis that consists of steatosis, hepatocyte ballooning and lobular inflammation with or without fibrosis. Compared with patients with simple steatosis, those with NASH are more likely to progress to cirrhosis, liver failure, or hepatocellular carcinoma. ${ }^{9}$ However, there are currently no established treatments for NASH other than lifestyle modification and weight loss, which are often difficult to achieve and even harder to maintain. ${ }^{10-12}$

A predominant feature of NAFLD is insulin resistance, the central mechanism in the development of hepatic steatosis. Insulin resistance leads to altered adipokine production and increased outflow of free fatty acids, resulting in hepatocellular accumulation of toxic lipid-derived metabolites and activation of multiple inflammatory pathways. ${ }^{13-15}$ Over time, this process can lead to activation of fibrogenic hepatic stellate cells. ${ }^{16}$ Therefore, several studies have explored the ability of insulin sensitizers to improve biochemical and histological features of fibrosis and inflammation in NAFLD. The two main groups of insulin-sensitizing drugs that have been evaluated for NASH treatment are thiazolidinediones and metformin. Thiazolidinediones (also known as the 'glitazones') are high-affinity ligands of peroxisome proliferator-activated receptor- $\gamma($ PPAR $-\gamma)$, that stimulate the storage of free fatty acids in subcutaneous adipocytes as opposed to liver and omental fat, thereby improving insulin sensitivity. ${ }^{17}$ Metformin is an insulin-sensitizer that acts by decreasing hepatic glucose production and increasing skeletal muscle glucose uptake.

Conclusions about the role of insulin-sensitizing agents such as thiazolidinediones (glitazones) or metformin for the treatment of NASH from currently available studies are limited due to small sample size, heterogeneous study endpoints and a range of methodological quality. The purpose of this meta-analysis is to summarize the currently available evidence for the efficacy of insulin-sensitizing agents on biochemical and histological endpoints in NASH.

\section{METHODS}

\section{Literature search}

A computer-assisted search was conducted to identify potentially relevant publications in the OVID MEDLINE database on 27 January 2010. The search (1997 to January 2010) was performed using the following exploded (exp) medical subject heading (MeSH), textwords, and truncated text words (\$): metformin, thiazolidinedione (exp), pioglitazone, rosiglitazone, insulin sensitiz $\$$, therapeutics (exp), treatment, fatty liver (exp), non-alcoholic fatty liver disease, non-alcoholic steatohepatitis, steatosis, NAFLD, NASH, liver. The preceding terms were combined with appropriate Boolean logic, and this search was then limited to humans and adults. A similar search was also performed in EMBASE, Pubmed and the Cochrane Central Register of Controlled Trials from 1997 to 2010. Additional electronic searches of Digestive Diseases Week (DDW) abstracts, American Association for Study Liver Diseases of (AASLD) meeting abstracts, American College of Gastroenterology (ACG) meeting abstracts and European Association for the Study of the Liver (EASL) meeting abstracts for the period from 2005 to 2009 were performed with combinations of the search terms 'fatty liver' or 'steatohepatitis' or 'NASH' and 'treatment'. A manual search of abstracts from these years was also performed. Manual recursive searches of references from review articles and published randomized controlled trials that met inclusion criteria were also completed. Finally, consultation with expert hepatologists was performed to identify any additional abstracts or unpublished data.

\section{Study selection criteria}

Potentially relevant studies were selected based on the following inclusion criteria: (i) randomized controlled trials using an insulin sensitizer drug that is currently FDA-approved (metformin, rosiglitazone, pioglitazone); (ii) diagnosis of NASH determined by histology; (iii) liver biopsy performed at beginning and end of clinical trial; and (iv) adult patients. Studies in which NASH was diagnosed based on elevated transaminases or abnormal imaging studies (ultrasound, CT scan, magnetic resonance imaging) without histological confirmation were excluded. Studies that involved weight loss therapies such as orlistat or bariatric surgery were not included because they primarily target weight loss and only secondarily affect insulin resistance. ${ }^{18}$

All randomized controlled trials, regardless of publication status, number of patients randomized, language, or blinding were included. Studies published only as abstracts 
were included if they had sufficient information on study design, characteristics of participants, interventions and outcomes and/or if an author of the abstract could provide this information. One investigator (MR) reviewed the titles and abstracts of all citations identified by the literature search. Potentially relevant studies were retrieved. Two investigators (MR, AS) independently applied the selection criteria, and any disagreements were resolved by consensus. Agreement between investigators for selection of studies for the meta-analysis was greater than $95 \%$.

\section{Data extraction}

Eligible articles were reviewed in a duplicate, independent manner by two investigators (MR, AS). For each study, the investigators collected the following data: (i) Study: year, location, design, publication status; (ii) Treatment: dose, frequency, duration, active diet and exercise education throughout trial; (iii) Patients: number, mean age, gender, presence of diabetes; (iv) Anthropometrics and laboratory tests (baseline and end of study): BMI, ALT; and (v) Histology (baseline and end of study): steatosis grade, hepatocyte ballooning score, lobular inflammation score and fibrosis stage. If data were not available in the published article, the investigators contacted the corresponding authors for additional information. Any discrepancies in data quantification were resolved by discussion among the investigators.

\section{Primary and secondary outcomes}

The primary outcomes for this meta-analysis were histological responses to treatment, specifically changes in: (i) steatosis grade (0-3), (ii) hepatocyte ballooning score (02 ), (iii) lobular inflammation score (0-3), or (iv) fibrosis stage $(0-4)$. The secondary outcomes included (i) biochemical response, measured as change in ALT and (ii) anthropometric response, measured as change in body mass index (BMI).

\section{Quality assessment}

Study quality was assessed using the Cochrane Collaboration's tool for assessing risk of bias. ${ }^{19}$ As opposed to other assessment tools that utilize scales or check lists, this tool utilizes domain-based evaluation, which focuses on criteria that are more highly associated with internal validity in RCTs. Quality assessment was based on the following domains: (i) randomization, (ii) allocation concealment, (iii) blinding of participants, personnel, and outcome assessors, (iv) completeness of outcome data, (v) unbiased outcome reporting and (vi) lack of other sources of bias. Studies were categorized as high quality if all six domains were well described and low quality if one or more domains were not well described.

\section{Statistical analysis}

The outcomes were calculated as mean difference in scores in the intervention group and were compared with the mean difference in scores in the control group, as described by the following equation: (treatment mean at baseline - treatment mean at the end of the study) (control mean at baseline - control mean at the end of the study). Therefore, if the histological response to treatment improved to a greater extent in the treatment compared with the control group, the weighted mean difference (WMD) would be positive. Likewise, if the response improved to a greater extent in the control group compared with the treatment group, the WMD would be negative. Difference in means for each outcome for intervention and control group was either directly obtained from the study results or calculated by determining the difference between reported mean values before and after treatment. However, studies did not report the s.d. of the change in means, but reported only the s.d. of the preintervention mean and the s.d. of the postintervention mean. One study provided the s.d. of the change in means for all outcomes upon request. ${ }^{25}$ For those studies that reported a $P$-value for the comparison between pre- and postintervention mean values, ${ }^{26-28,33}$ a s.d. for the change in means was derived through backcalculation. For those studies without $P$-values, $t$-statistics, or confidence intervals, ${ }^{30-32}$ we imputed the s.d. of the change in means, using a modified method by Follmann et al.: s.d. $_{\text {change in means }}=$ sq root $\left[\left(\text { s.d. }_{\text {pre }}\right)^{2}+(\text { s.d. } \text { post })^{2}-\right.$ $\left(2(\rho) \times\right.$ s.d.pre $_{\text {. }} \times$ s.d.post $\left._{\text {pos }}\right){ }^{20}$ This equation estimates the

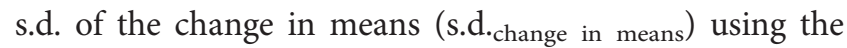
s.d. of preintervention mean (s.d.pre) and the s.d. of postintervention mean (s.d.post) as well the within-participant correlation $(\rho)$ of the outcome measure. We initially assumed a within-participant correlation of zero to derive the largest standard deviation of the change in means, which thereby calculates the most conservative measurement of pooled s.d. for this meta-analysis. A subsequent sensitivity analysis was performed using varied measurements of $\rho(\rho=0.4, \rho=0.8)$ to evaluate if varied levels of within-participant correlation altered the statistical significance for each outcome.

Estimates of effect, WMD, were pooled using the DerSimonian and Laird method for a random effects model. For each outcome variable, a forest plot was created that illustrates both study-specific and pooled WMD with $95 \%$ confidence intervals. Heterogeneity was 
assessed using the Cochrane $Q$ statistic and the inconsistency index $\left(I^{2}\right){ }^{21,22} \mathrm{~A}$ chi-square $P$-value $<0.05$ or $I^{2}$ $>50 \%$ is consistent with the possibility of substantial heterogeneity. ${ }^{22}$ Influence analysis, in which one study is removed at a time from the model, was performed to determine if there was a possible undue influence of a single study. ${ }^{23}$ Publication bias was graphically examined using a symmetric funnel plot, and then statistically using the test of Egger. ${ }^{24} \mathrm{~A}$ contour-enhanced symmetric funnel plot helps to rule out bias due to small studies that were not published due to unfavourable results. All statistics were computed using STATA 11.0 (StataCorp LP, College Station, TX, USA).

Subset analysis was performed for predefined subsets of studies based on (i) type of insulin sensitizer, (ii) exclusion of patients with diabetes, (iii) use of concurrent diet and exercise, (iv) inclusion of patients with benign steatosis in addition to patients with NASH, (v) length of follow-up and (vi) study quality.

\section{RESULTS}

\section{Literature search}

A total of 185 citations were retrieved using our search strategy, and all citations were downloaded into EndNote.
Search of abstracts from the annual meetings of American Association for Study of Liver Diseases (AASLD), American College of Gastroenterology (ACG), European Association for the Study of the Liver (EASL) and Digestive Disease Week (DDW) for the period from 20052009 yielded 12 potentially relevant abstracts. Recursive searches of article references identified three additional articles. After review of the titles and abstracts of all of these articles $(n=200), 181$ were excluded. Nineteen studies underwent full text review to determine their eligibility. Of these, two were initially in abstract form. A full manuscript was provided by one author after being contacted for more information. The other abstract was published soon after abstract presentation and therefore full manuscript was included in this meta-analysis. After review of the 19 full text articles, a total of nine studies were included in this meta-analysis (Figure 1, Table 1). ${ }^{25-33}$ Although important as the original study that investigated the use of insulin sensitizers for the management of NAFLD, we excluded Caldwell's important trial involving troglitazone because this trial was not a randomized controlled trial and therefore did not meet our inclusion criteria. ${ }^{34}$ There did not appear to be any publication bias by Egger's test $(P=0.73)$ or funnel plot analysis (data not shown).

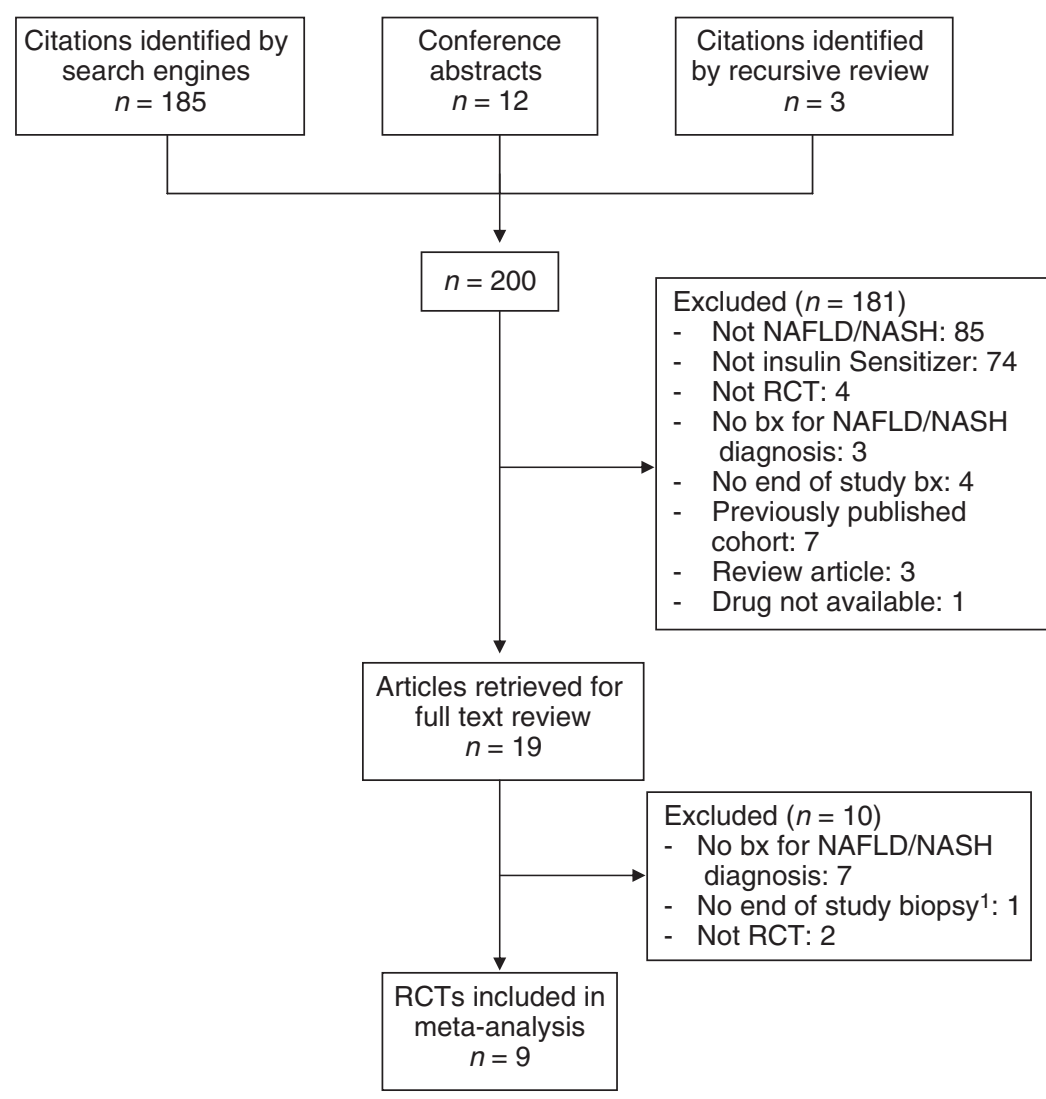

${ }^{1}$ End of study biopsy was available for the control, but not for the treatment group
Figure 1 | Map of the literature search and selection process. $\mathrm{RCT}$, randomized controlled study; bx, biopsy. 


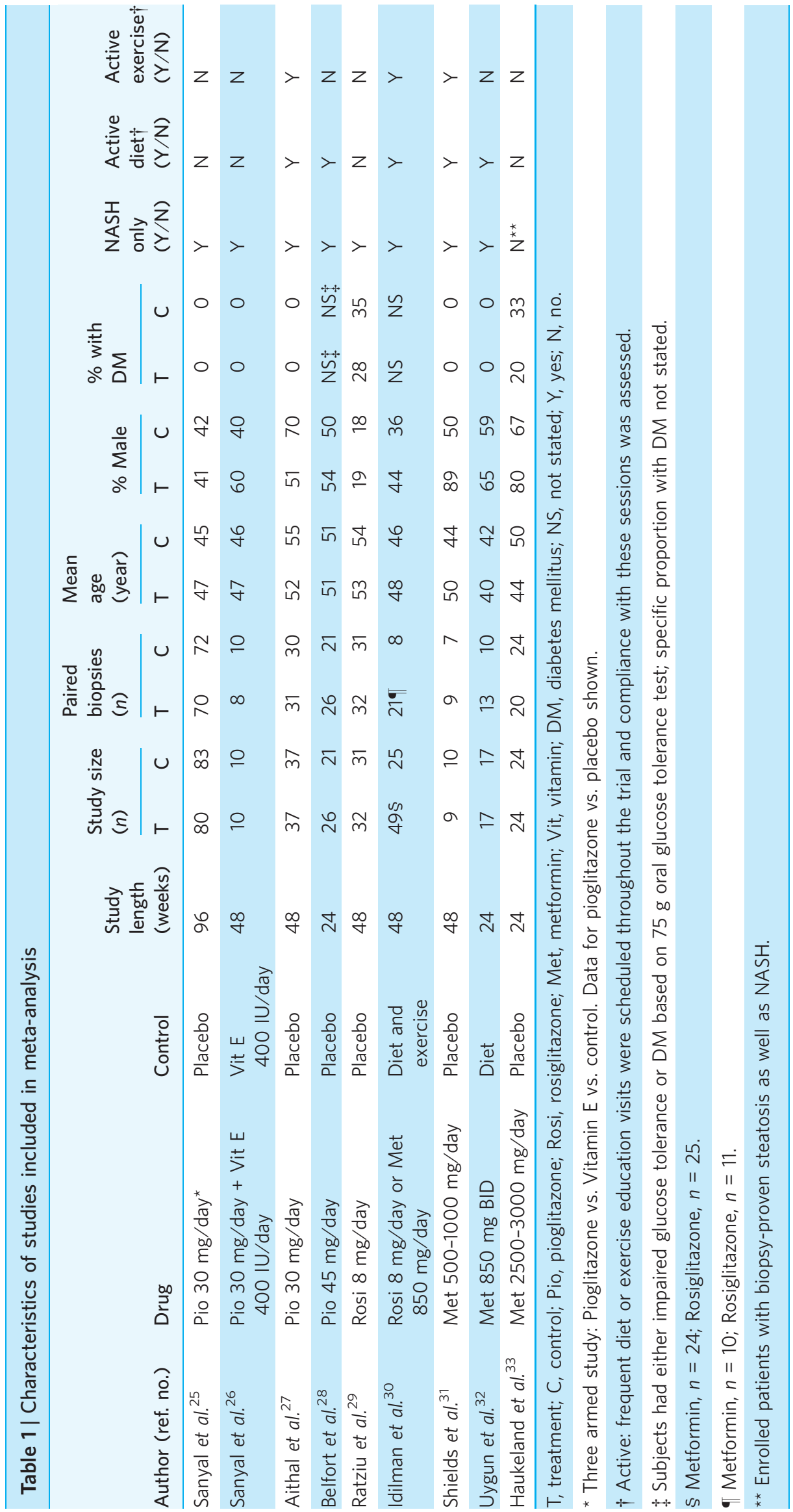




\section{Study description}

Of the nine trials included in our meta-analysis, six investigated the use of glitazones. ${ }^{25-30}$ These studies used either pioglitazone or rosiglitazone, with a treatment duration of 96 weeks, ${ }^{25} 48$ weeks, ${ }^{26,27,29,30}$ or 24 weeks. ${ }^{28}$ All six trials excluded patients with benign steatosis and enrolled only patients with NASH. Of note, Idilman et al. had three arms (rosiglitazone, metformin, control); therefore, this study was included in subgroup analysis for both glitazones and metformin. ${ }^{30}$

Four trials investigated the use of metformin ${ }^{30-33}$ using dosages ranging from $500 \mathrm{mg}^{31}$ to $3000 \mathrm{mg}^{33}$ daily. Duration of treatment was 24 weeks in two studies ${ }^{32,33}$ and 48 weeks in the other two. ${ }^{30,31}$ All trials enrolled only patients with NASH except one which also included patients with simple steatosis. ${ }^{33}$ One trial ${ }^{32}$ did not report results of histological outcomes other than fibrosis and therefore only three trials ${ }^{30,31,33}$ were included for analysis of steatosis, hepatocyte ballooning and lobular inflammation.

\section{Outcomes}

Primary outcome: histological response. Table 2 summarizes the pooled treatment effect WMD (weighted mean difference) of insulin sensitizers for all analyses. Analysing all nine studies together, insulin sensitizing agents resulted in a significant improvement in fibrosis $(P=0.011)$ and steatosis $(P=0.003)$ compared with controls, but not ballooning $(P=0.10)$ or inflammation $(P=0.29)$.

In subgroup analysis, glitazones resulted in a significant reduction in steatosis $(P<0.001)$ and hepatocyte ballooning $(P<0.001)$ compared with controls (Table 2 ,
Figure 2). Glitazones did not result in improvement in lobular inflammation $(P=0.09)$ or fibrosis $(P=0.11)$ compared with the control group. Metformin did not improve pooled treatment effects for any of the histological outcomes (Table 2); specifically, there was no associated improvement in fibrosis $(P=0.51)$.

Secondary outcomes: biochemical and anthropometric response. When analysing all included studies, insulin sensitizing agents did result in improvement in ALT $(P=0.014)$ compared with controls (Table 2). Glitazones resulted in a highly significant decrease in ALT compared with controls $(P<0.001)$, whereas metformin did not improve pooled treatment effects for biochemical outcome $(P=0.10)$.

Overall, BMI improved (i.e. decreased from baseline) to a greater extent in the control group than in the group receiving insulin sensitizers $\quad(\mathrm{WMD}=-1.23$; $P<0.001$ ). In particular, BMI improved in the controls compared with the patients receiving glitazones only $(P=0.010)$, whereas metformin use did not result in significant change in BMI compared with the control group $(P=0.39)$ (Table 2).

\section{Sensitivity analyses}

As described previously, variance imputation was initially performed using a within-participant correlation of zero $(\rho=0.0)$. Sensitivity analysis using other values of $\rho$ ( $\rho=0.4, \rho=0.8$ ) revealed no difference in trends for each outcome, yielding similar findings for all statistical analyses (data not shown).

Table 2 | Summary of effect sizes (weighted mean difference) for all insulin sensitizers, glitazones and metformin compared with controls

\begin{tabular}{|c|c|c|c|c|c|c|c|c|c|}
\hline \multirow[b]{2}{*}{ Outcomes } & \multicolumn{3}{|c|}{ All insulin sensitizers } & \multicolumn{3}{|c|}{ Glitazones } & \multicolumn{3}{|c|}{ Metformin } \\
\hline & WMD* $^{\star}$ & $95 \% \mathrm{Cl}$ & $P$-value & WMD* $^{\star}$ & $95 \% \mathrm{Cl}$ & $P$-value & $W_{M D}^{\star}$ & $95 \% \mathrm{Cl}$ & $P$-value \\
\hline \multicolumn{10}{|c|}{ Primary outcome: histological response } \\
\hline Steatosis & 0.40 & $0.14,0.65$ & 0.003 & 0.57 & $0.36,0.77$ & $<0.001$ & -0.19 & $-0.69,0.31$ & 0.45 \\
\hline Ballooning & 0.16 & $-0.031,0.35$ & 0.10 & 0.36 & $0.24,0.49$ & $<0.001$ & -0.037 & $-0.19,0.12$ & 0.64 \\
\hline Inflammation & 0.17 & $-0.15,0.48$ & 0.29 & 0.29 & $-0.05,0.63$ & 0.09 & -0.19 & $-0.55,0.17$ & 0.31 \\
\hline Fibrosis & 0.24 & $0.053,0.42$ & 0.011 & 0.21 & $-0.046,0.46$ & 0.11 & 0.22 & $-0.37,0.81$ & 0.46 \\
\hline \multicolumn{10}{|c|}{ Secondary outcome: biochemical and anthropometric response } \\
\hline ALT & 11.9 & $2.4,21.5$ & 0.004 & 16.4 & $7.70,25.0$ & $<0.001$ & 13.6 & $-2.7,29.9$ & 0.10 \\
\hline $\mathrm{BMI}$ & -1.23 & $-1.61,-0.85$ & $<0.001$ & -0.90 & $-1.59,-0.22$ & 0.010 & 0.75 & $-0.97,2.48$ & 0.39 \\
\hline
\end{tabular}

WMD, weighted mean difference; $\mathrm{Cl}$, confidence interval; DM, diabetes mellitus; ALT, alanine aminotransferase; BMI, body mass index.

*WMD: a positive WMD indicates greater improvement in the treatment group compared with controls. 

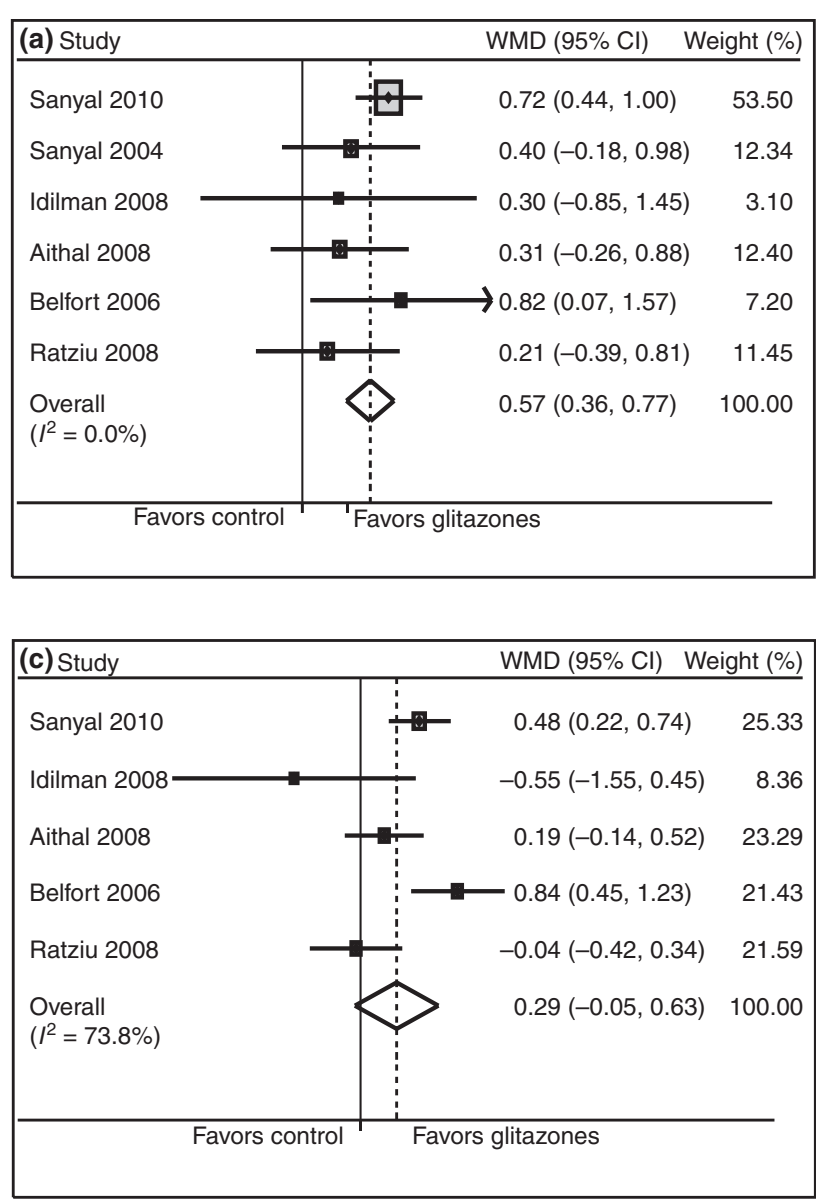
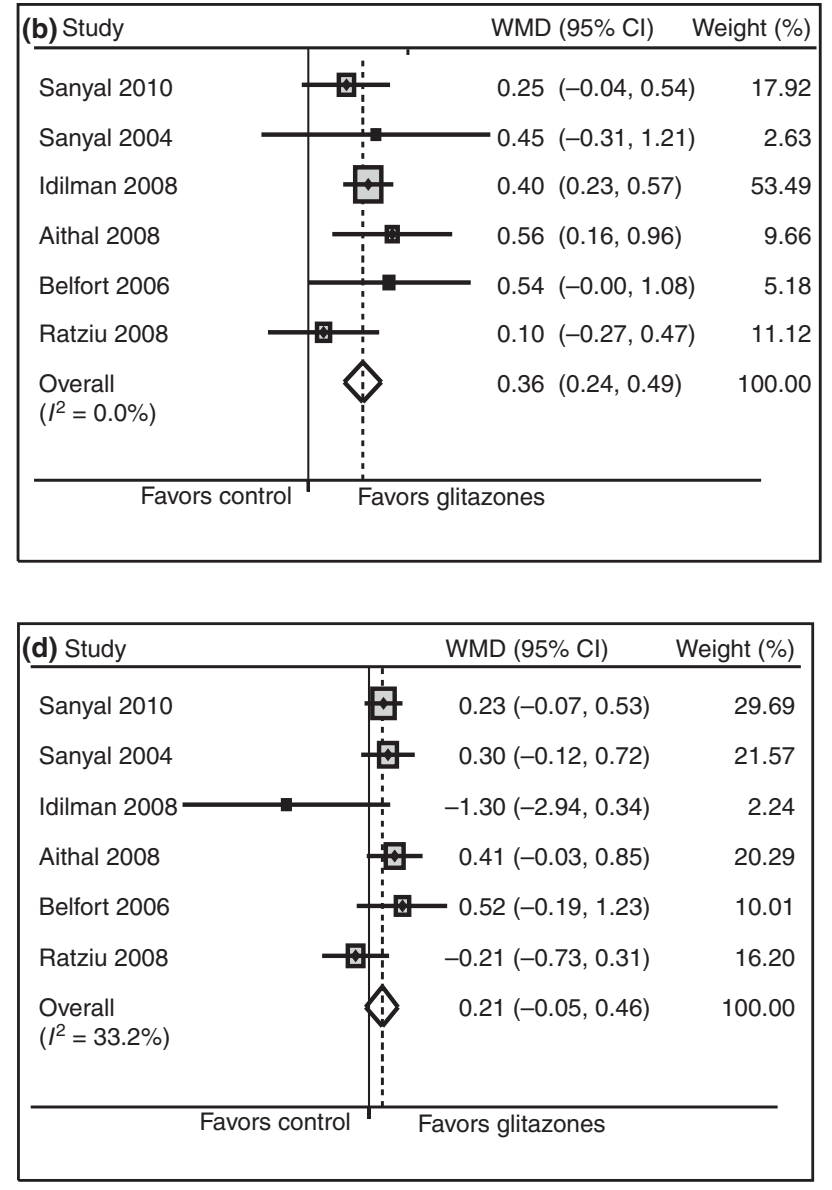

Figure 2 | Forest plots demonstrating pooled effect of glitazones on histological outcomes: a. steatosis, b. hepatocyte ballooning, c. inflammation and d. fibrosis.

An additional sensitivity analysis was performed to assess any effect of study quality. Four of the nine trials were considered low quality, ${ }^{26,30-32}$ primarily due to inadequate descriptions of allocation concealment or blinding, whereas the others were considered high quality $^{25,27-29,} 33$ (Table S1). At the time of baseline and end of study biopsies, pathologists in all studies were blinded to randomization. Intention-to-treat analysis was used in all but two trials. ${ }^{27,} 30$

Other preplanned subset analyses failed to affect significantly any of our primary or secondary outcomes. There was only one study ${ }^{33}$ that enrolled patients with simple steatosis; in addition, those with NASH and removal of this study did not affect overall outcomes. Active diet and/or exercise education during the clinical trial $^{27,28,30,31}$ did not affect outcomes, nor did study duration.

Sensitivity analyses for glitazone trials. Given the large $I^{2}$ for one of the primary outcomes in the glitazone trials (inflammation, $I^{2}=73.8 \%$ ), additional sensitivity analyses were performed for the glitazone subset. The first assessed undue influence from the PIVENS trial, the largest RCT for NASH treatment to date. Exclusion of the PIVENS data did not change the primary outcomes of our metaanalysis (Table 3). Specifically, after excluding the PIVENS trial from analysis, glitazones continued to demonstrate a significant improvement in steatosis $(P=0.01)$ and ballooning $(P<0.001)$ compared with controls, but not inflammation $(P=0.39)$ and fibrosis $(P=0.33)$ (Table 3$)$.

An additional sensitivity analysis was performed to assess any change in pooled outcomes related to study quality. When including only high quality studies, ${ }^{25,}$ 27-29 significance of primary and secondary outcomes remained the same, except that a significant decrease in inflammation was found (WMD $=0.37 ; 95 \%$ CI $0.04-$ $0.70 ; P=0.03$ ) (Table 3).

The final glitazone sensitivity analysis assessed trials that specifically excluded patients with diabetes. ${ }^{25-27}$ All three trials used pioglitazone at a dose of $30 \mathrm{mg}$ /day for 
M. O. Rakoski et al.

Table 3 | Sensitivity analyses for glitazones

Excluding PIVENS ${ }^{25}$

Outcomes
High quality studies only $25,27-29$

$P$-value $P^{2} \dagger W^{*} D^{*} 95 \% \mathrm{Cl}$
$P$-value
Non-DM trials only ${ }^{25-27}$

WMD $^{\star} 95 \% \mathrm{Cl} \quad P$-value $P^{2}+$

Primary outcome: histological response

$\begin{array}{lrrrrrrrrrrrr}\text { Steatosis } & 0.39 & 0.09,0.68 & 0.01 & 0 & 0.57 & 0.30,0.84 & <0.001 & 18 & 0.59 & 0.34,0.84 & <0.001 & 8 \\ \text { Ballooning } & 0.39 & 0.25,0.52 & <0.001 & 0 & 0.32 & 0.11,0.53 & 0.002 & 16 & 0.37 & 0.14,0.59 & 0.001 & 0 \\ \text { Inflammation } & 0.21 & -0.27,0.68 & 0.39 & 78 & 0.37 & 0.04,0.70 & 0.03 & 75 & 0.35 & 0.071,0.64 & 0.014 & 46 \\ \text { Fibrosis } & 0.18 & -0.18,0.54 & 0.33 & 46 & 0.22 & -0.04,0.49 & 0.10 & 25 & 0.29 & 0.078,0.51 & 0.008 & 0\end{array}$

Secondary outcome: biochemical and anthropometric response

$\begin{array}{lcccccccccccc}\text { ALT } & 12.0 & -0.70,24.7 & 0.06 & 27 & 20.7 & 19.2,22.1 & <0.001 & 0 & 19.2 & 5.11,33.2 & 0.008 & 47 \\ \text { BMI } & -0.59 & -1.27,0.09 & 0.09 & 14 & -1.39 & -1.48,-1.30 & <0.001 & 0 & -1.23 & -1.9,-0.57 & <0.001 & 14\end{array}$

WMD, weighted mean difference; $\mathrm{Cl}$, confidence interval; $l^{2}$, inconsistency index; $\mathrm{DM}$, diabetes mellitus; $\mathrm{ALT}$, alanine aminotransferase; BMI, body mass index.

* WMD: a positive WMD indicates greater improvement in the treatment group compared with controls.

$\dagger P^{2}(\%)$ is a summary statistic for evaluation of heterogeneity across studies. $I^{2}>50 \%$ suggest the possibility of substantial heterogeneity.
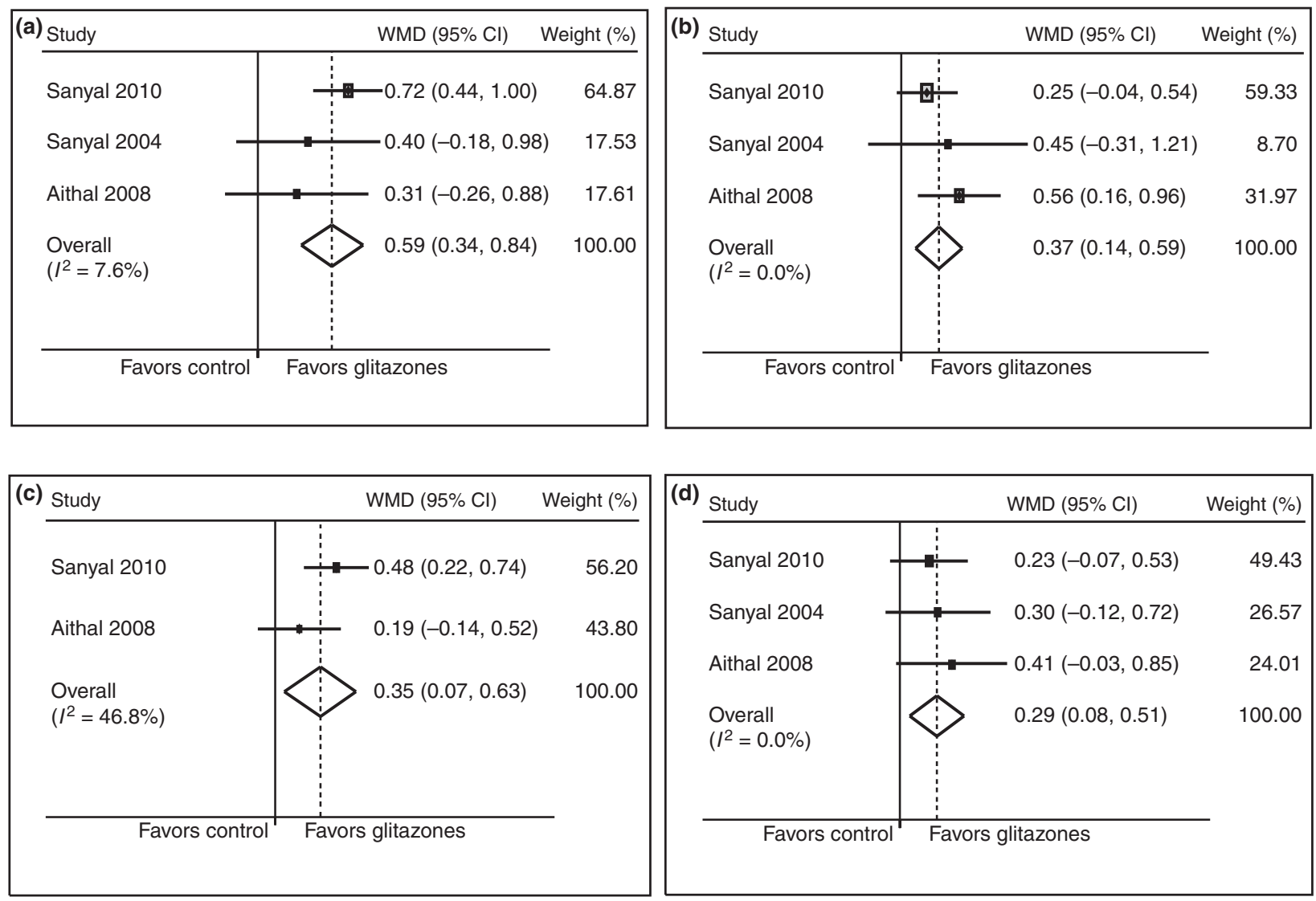

Figure 3 | Forest plots demonstrating pooled effects of glitazones on histological outcomes in trials that excluded patients with diabetes: (a) steatosis, (b) hepatocyte ballooning, (c) inflammation and (d) fibrosis. 
a minimum duration of 48 weeks. When patients with diabetes were excluded, pioglitazone resulted in a significant decrease in all histological and biochemical outcomes, including fibrosis (WMD $=0.29$; 95\% CI 0.078 $0.51 ; P=0.008$ ) (Figure 3, Table 3). In addition, there was a significant increase in BMI $(P<0.001)$.

\section{DISCUSSION}

To date, conclusions about the efficacy and safety of insulin sensitizers for the treatment of NASH have been difficult due to the small sample size and heterogeneity of available trials. Our study is the largest and most comprehensive meta-analysis of randomized controlled trials evaluating the role of glitazones and metformin in these patients. We found that glitazones significantly improved steatosis, hepatocyte ballooning and serum ALT levels compared with controls, but not lobular inflammation or fibrosis. However, in patients without diabetes, glitazones resulted in significant decreases in all histological outcomes, including fibrosis. In contrast, metformin was found to be an ineffective treatment for $\mathrm{NASH}$ as it was not superior to controls for any histological or biochemical outcome.

Given that glitazones have potential serious side effects $^{35}$ and will probably need to be taken longterm, ${ }^{36,37}$ it is important to determine which subset of patients, if any, would most benefit from prolonged glitazone therapy. This meta-analysis offers novel insights into tailoring NASH therapy and suggests that glitazone treatment should be focused on patients who have not yet developed diabetes. Pooled data from trials that enrolled only patients without diabetes ${ }^{25-27}$ demonstrated a significant decrease in all biochemical and histological outcomes, including fibrosis $(P=0.008)$, the endpoint ultimately thought to be the most important in NASH treatment in preventing disease progression. Furthermore, Ratziu et al. found that absence of diabetes was an independent predictor of histological response in NASH patients treated with rosiglitazone $(\mathrm{OR}=0.14,95 \% \mathrm{CI}$ $0.02-0.79, P=0.026){ }^{29}$ Together, these findings suggest that patients without overt diabetes may be particularly susceptible to the insulin sensitizing properties of glitazones and that early intervention may prevent worsening of insulin resistance, as well as its downstream complications such as NASH progression.

There are several potential mechanisms to explain the differential benefit of glitazones on inflammation and fibrosis in patients with and without diabetes. First, glitazones may prevent the development of insulin resistance by preservation of pancreatic beta cell function and by increasing the effectiveness of endogenous insulin, as seen in studies of high-risk populations such as patients with impaired glucose tolerance (IGT), impaired fasting glucose (IFG), or a history of gestational diabetes. ${ }^{38-40}$ Specifically, the DREAM trial, a large, multicentre trial that randomized 5269 patients with IFG or IGT to receive rosiglitazone or placebo for a median of 3 years found that rosiglitazone reduced the risk of developing diabetes or death by $60 \%$ compared with placebo, with an absolute risk difference of $14.4 \%{ }^{38}$ Our findings suggest that glitazones may arrest NASH progression, perhaps through a similar mechanism of delayed or halted development of insulin resistance and/or preventing worsening of insulin resistance in those who do not already have overt diabetes. Second, any improvement in fibrosis from glitazone use is probably indirect and mediated through anti-inflammatory pathways given that PPAR-gamma is minimally expressed in activated hepatic stellate cells. ${ }^{41}$ Therefore, in diabetics, the potent antiinflammatory effects of glitazones ${ }^{42}$ may be surpassed by the pro-inflammatory state associated with insulin resistance state and diabetes, ${ }^{43}$ thereby potentially making this class of drugs less effective for NASH treatment among patients with diabetes.

In the recently published PIVENS trial, ${ }^{25}$ pioglitazone failed to meet the composite primary endpoint, which required a decrease in NAFLD Activity Score (NAS) ${ }^{44}$ of at least two points with at least one point decrease in ballooning and no worsening of fibrosis $(P=0.04$; $P<0.025$ considered significant due to three-arm design). This trial was a three-arm randomized controlled trial comparing 96 weeks of pioglitazone, vitamin $\mathrm{E}$, and placebo for treatment of nondiabetic patients with NASH. However, when each histological component that comprises the NAS was assessed individually, pioglitazone did result in a significant decrease in steatosis, hepatocyte ballooning and lobular inflammation compared with controls $(P<0.001,0.01$ and $<0.001$ respectively). A trend towards greater mean decrease in fibrosis (week 96 fibrosis stage -baseline fibrosis stage) was found for pioglitazone compared with controls $(-0.36 \pm 0.96$ and $0.13 \pm 0.89$ respectively); however, it did not reach statistical significance $(P=0.10)$. Amongst the trials that enrolled only nondiabetic patients including the PIVENS study, ${ }^{25-27}$ our meta-analysis found a significant decrease in fibrosis with glitazone therapy compared with controls in patients without diabetes $(P=0.008)$ with minimal heterogeneity between trials for this outcome $\left(I^{2}=0 \%\right)$. Although the PIVENS trial is the largest randomized controlled trial to date, change in fibrosis was only a 
secondary endpoint and therefore it is likely that the trial may have been underpowered to detect a difference of this magnitude. Our meta-analysis suggests that glitazone therapy may improve fibrosis for select populations such as those without diabetes.

This meta-analysis has a few limitations that warrant discussion. First, selection and referral bias should be considered given that all of the included trials were conducted in tertiary care centres. Second, our method for weighting studies made assumptions that resulted in the largest variance for the pooled effect size. Therefore, it is possible that 95\% confidence intervals for the WMD are wider and more conservative than what would be expected if the within-person correlations were known. However, sensitivity analyses did not alter the statistical significance for each outcome, thereby demonstrating the robustness of the findings. Third, the NASH Clinical Research Network (NASH CRN) recently created a comprehensive scoring system for clinical trials, the NAFLD Activity Score (NAS); ${ }^{44}$ however, not all trials reported this score and therefore we were not able to use the NAS as an endpoint in our analysis. Finally, as with all studies that utilize histological endpoints, our findings are limited by the inherent sampling variability with resultant inaccuracies sometimes associated with liver biopsies. We feel that the notable strengths of this meta-analysis, including the strict inclusion criteria (RCTs with a histological definition of NAFLD/NASH) and the assessment of both histological and biochemical outcomes, outweigh its limitations.

In summary, it is evident from our findings that whereas metformin is not an effective treatment for $\mathrm{NASH}$, the use of glitazones, on the other hand, results in a significant biochemical improvement as well as decrease in important histological components of NASH such as steatosis and hepatocyte ballooning, but not lobular inflammation or fibrosis. In patients without diabetes, glitazones produce a significant improvement in all biochemical and histological outcomes, most significantly including fibrosis. Despite this novel finding, there is insufficient evidence at this time to make a strong recommendation for the use of glitazones in patients without diabetes. Future studies are needed to investigate the outcomes and potential benefits and toxicities of longterm glitazone treatment in this subgroup of patients with NASH. Moreover, additional studies in nondiabetics are also needed to determine if glitazones offer incremental benefit over anti-oxidants such as vitamin E, which may be equally efficacious or more efficacious, ${ }^{25}$ without detrimental side effects such as weight gain.

\section{ACKNOWLEDGEMENT}

Declaration of personal and funding interests: None.

\section{SUPPORTING INFORMATION}

Additional Supporting Information may be found in the online version of this article:

Table S1. Quality assessment using the Cochrane Collaboration's tool for assessing risk of bias ( $\mathrm{Y}=$ yes, $\mathrm{U}=$ unclear, $\mathrm{N}=$ no).

Please note: Wiley-Blackwell are not responsible for the content or functionality of any supporting materials supplied by authors. Any queries (other than missing material) should be directed to the corresponding author for the article.

\section{REFERENCES}

1. Bellentani S, Saccoccio G, Masutti F, et al. Prevalence of and risk factors for hepatic steatosis in northern Italy. Ann Intern Med 2000; 132: 112-7.

2. Adams LA, Harmsen S, St Sauver JL, et al. Nonalcoholic fatty liver disease increases risk of death among patients with diabetes: a community-based cohort study. Am J Gastroenterol 2010; 105: 1567-73.

3. Younossi ZM, Gramlich T, Matteoni CA, Boparai N, McCullough AJ. Nonalcoholic fatty liver disease in patients with type 2 diabetes. Clin Gastroenterol Hepatol 2004; 2: 262-5.

4. Haynes P, Liangpunsakul S, Chalasani N. Nonalcoholic fatty liver disease in individuals with severe obesity. Clin Liver Dis 2004; 8: 535-47.

5. Ekstedt M, Franzén LE, Mathiesen UL, et al. Long-term follow-up of patients with NAFLD and elevated liver enzymes. Hepatology 2006; 44: 86573.

6. Ong JP, Pitts A, Younossi ZM. Increased overall mortality and liver-related mortality in non-alcoholic fatty liver disease. J Hepatol 2008; 49: 608-12.

7. Edens MA, Kuipers F, Stolk RP. Nonalcoholic fatty liver disease is associated with cardiovascular disease risk markers. Obesity reviews 2009; 10: 412-9.

8. Picardi A, Vespasiani-Gentilucci U. Association between non-alcoholic fatty lvier disease and cardiovascular disease: a first message should pass. Am J Gastroenterol 2008; 103: 3036-8.

9. Bellentani S, Marino M. Epidemiology and natural history of non-alcoholic fatty liver disease (NAFLD). Ann Hepatol 2009; 8(Suppl 1): S4-8.

10. Ratziu V, Zelber-Sagi S. Pharmacologic therapy of non-alcoholic steatohepatitis. Clin Liver Dis 2009; 13: 667-88.

11. Promrat K, Kleiner DE, Niemeier HM, et al. Randomized controlled trial testing the effects of weight loss on nonalcoholic steatohepatitis (NASH). Hepatology 2010; 51: 121-9.

12. Huang MA, Greenson JK, Chao C, et al. One-year intense nutritional counseling 
results in histological improvement in patients with non-alcoholic steatohepatitis: a pilot study. Am J Gastroenterol 2005; 100: 1072-81.

13. Cusi K. Role of insulin resistance and lipotoxicity in non-alcoholic steatohepatitis. Clin Liver Dis 2009; 13: 545-63.

14. Gentile CL, Pagliassotti MJ. The role of fatty acids in the development and progression of nonalcoholic fatty liver disease. J Nutr Biochem 2008; 19: 567-76.

15. Oh MK, Winn J, Poordad F. Review article: diagnosis and treatment of nonalcoholic fatty liver disease. Aliment Pharmacol Ther 2008; 28: 503-22.

16. Tiniakos DG, Vos MB, Brunt EM. Nonalcoholic fatty liver disease: pathology and pathogenesis. Annu Rev Pathol 2010; 5: 145-71.

17. Shulman GI. Cellular mechanisms of insulin resistance. J Clin Invest 2000; 106: 171-6.

18. Bray GA. Medications for obesity: mechanisms and applications. Clin Chest Med 2009; 30: 525-38.

19. Higgins JPT, Green S (editors). Cochrane handbook for systematic reviews of interventions version 5.0.2. The Cochrane Collaboration, 2009. Available at: http://www.cochrane-handbook.org.

20. Follmann D, Elliott P, Suh I, Cutler J. Variance imputation for overviews of clinical trials with continuous response. J Clin Epidemiol 1992; 45: 769-73.

21. Cochran W. The combination of estimated from different experiments. Biometrics 1954; 10: 101-29.

22. Higgins JPT, Thompson SG. Quantifying heterogeneity in a meta-analysis. Statist Med 2002; 21: 1539-58.

23. Copas J, Shi JQ. Meta-analysis, funnel plots and sensitivity analysis. Biostatistics 2000; 1: 247-62.

24. Egger M, Davey Smith G, Schneider M, Minder C. Bias in meta-analysis detected by a simple graphical test. BMJ 1996; 315: 629-34.

25. Sanyal AJ, Chalasani N, Kowdley KV, et al. Pioglitazone, vitamin E, or placebo for nonalcoholic steatohepatitis. New Engl J Med 2010; 362: 1675-85.

26. Sanyal AJ, Mofrad PS, Contos MJ, et al. A pilot study of vitamin $\mathrm{E}$ versus vitamin $\mathrm{E}$ and pioglitazone for the treatment of nonalcoholic steatohepatitis. Clin Gastroenterol Hepatol 2004; 2: 1107-15.

27. Aithal GP, Thomas JA, Kaye PV, et al. Randomized, placebo-controlled trial of pioglitazone in nondiabetic subjects with nonalcoholic steatohepatitis. Gastroenterology 2008; 135: 1176-84.

28. Belfort R, Harrison SA, Brown $\mathrm{K}$, et al. A placebo-controlled trial of pioglitazone in subjects with nonalcoholic steatohepatitis. N Engl J Med 2006; 355: 2297-307.

29. Ratziu V, Giral P, Jacqueminet $S$, et al. Rosiglitazone for nonalcoholic steatohepatitis: one-year results of the randomized placebo-controlled Fatty Liver Improvement with Rosiglitazone Therapy (FLIRT) Trial. Gastroenterology 2008; 135: $100-10$.

30. Idilman R, Mizrak D, Corapcioglu D, et al. Clinical trial: insulin-sensitizing agents may reduce consequences of insulin resistance in individuals with nonalcoholic steatohepatitis. Aliment Pharmacol Ther 2008; 28: 200-8.

31. Shields WW, Thompson KE, Grice GA, Harrison SA, Coyle WJ. The effect of metformin and standard therapy versus standard therapy alone in nondiabetic patients with insulin resistance and nonalcoholic steatohepatitis (NASH): a pilot study. Therap Adv Gastroenterol 2009; 2: 157-63.

32. Uygun A, Kadayifci A, Isik AT, et al. Metformin in the treatment of patients with non-alcoholic steatohepatitis. Aliment Pharmacol Ther 2004; 19: 537-44.

33. Haukeland JW, Konopski Z, Eggesbø $\mathrm{HB}$, et al. Metformin in patients with non-alcoholic fatty liver disease: a randomized, controlled trial. Scand J Gastroenterol 2009; 44: 853-60.

34. Caldwell SH, Hespenheide EE, Redick JA, Iezzoni JC, Battle EH, Sheppard BL. A pilot study of a thiazolidinedione, troglitazone, in nonalcoholic steatohepatitis. Am J Gastroenterol 2001; 96: 519-25.

35. Kaul S, Bolger AF, Herrington D, Giugliano RP, Eckel RH. Thiazolidinedione drugs and cardiovascular risks: a science advisory from the American Heart Association and American College of
Cardiology Foundation. Circulation 2010; 121: 1868-77.

36. Lutchman G, Modi A, Kleiner DE, et al. The effects of discontinuing pioglitazone in patients with nonalcoholic steatohepatitis. Hepatology 2007; 46: 424-9.

37. Ratziu V, Charlotte F, Bernhardt C, et al. Long-term efficacy of rosiglitazone in nonalcoholic steatohepatitis: results of the fatty liver improvement by rosiglitazone therapy (FLIRT 2) extension trial. Hepatology 2010; 51: 445-53.

38. DREAM (Diabetes REduction Assessment with ramipril and rosiglitazone Medication) Trial Investigators, Gerstein HC, Yusuf S, et al. Effect of rosiglitazone on the frequency of diabetes in patients with impaired glucose tolerance or impaired fasting glucose: a randomised controlled trial. Lancet 2006; 368 : 1096-105.

39. Durbin RJ. Thiazolidinedione therapy in the preventin.delay of type 2 diabetes in patients with impaired glucose tolerance and insulin resistance. Diabetes Obes Metab 2004; 6: 280-5.

40. Buchanan TA, Xiang AH, Peters RK, et al. Preservation of pancreatic beta-cell function and prevention of type 2 diabetes by pharmacological treatment of insulin resistance in high-risk hispanic women. Diabetes 2002; 51: 2796-803.

41. Marra F, Efsen E, Romanelli RG, et al. Ligands of peroxisome proliferatoractivated receptor gamma modulate profibrogenic and proinflammatory actions in hepatic stellate cells. Gastroenterology 2000; 119: 466-78.

42. Mohanty P, Aljada A, Ghanim H, et al. Evidence for a potent anti-inflammatory effect of rosiglitazone. J Clin Endocrinol Metab 2004; 89: 2728-35.

43. Garg R, Tripathy D, Dandona P. Insulin resistance as a proinflammatory state: mechanisms, mediators, and therapeutic interventions. Curr Drug Targets 2003; 4: 487-92.

44. Kleiner DE, Brunt EM, Van Natta M, et al. Design and validation of a histological scoring system for nonalcoholic fatty liver disease. Hepatology 2005; 41: 1313-21. 